\title{
„You är ju även du“. Englisch als Mittel zur ,Selbst'-Reflexion in Aino Trosells En egen strand (2013)
}

\begin{abstract}
Der Beitrag untersucht Möglichkeiten des literarischen Einsatzes von Mehrsprachigkeit, die über die Funktion der Authentizitätsvergewisserung hinausgehen. Anhand des Romans En egen strand der schwedischen Autorin Aino Trosell (2013) wird die dortige Verwendung von Englisch als intertextueller Verweis gelesen. Der Roman handelt von dem Versuch einer Schwedin, in einem nicht näher lokalisierten pazifischen Inselarchipel sich eine neue Existenz als Aussteigerin und Künstlerin aufzubauen. Brigitta Buschs (2013) soziolinguistischer Gedanke, dass Mehrsprachigkeit Zugang zu „sprachlichen und kommunikativen Ressourcen“ bedeutet, ist Ausgangspunkt für das close reading des Romans. Die Analyse zeigt, wie der Roman zum einen über privilegierte Ressourcenzugänge durch den anglophonen Kolonialismus reflektiert und zum anderen eine feministische Kritik an der schwedischen Gesellschaft auf Grundlage von Virginia Woolfs englischsprachigem Werk formuliert. Die für das monolinguale Paradigma typische Homologie von Sprache, Ethnizität, Kultur und Nation (vgl. Yildiz 2011) wird in En egen strand durch die intertextuellen Verweise auf englischsprachige Traditionen des Kolonialismus und Feminismus gestört.
\end{abstract}

Keywords: Mehrsprachigkeit; Intertextualität; monolinguales Paradigma; Aino Trosell

Wer ist heutzutage noch einsprachig? Jedem Individuum steht eine Vielfalt an „sprachlichen und kommunikativen Ressourcen“ zur Verfügung, wie die Soziolinguistin Brigitta Busch $(2013,10)$ in ihrer Einführung zum Thema Mehrsprachigkeit feststellt. Angesichts dieser potenziellen Ressourcenvielfalt empfiehlt Busch aus linguistischer Sicht, von einer Heteroglossie im Sinne des Literatur- und Kulturtheoretikers Michail Bachtin auszugehen. Mit Bachtin ließen sich Sprachen weniger als geschlossene Systeme, sondern vielmehr als Konglomerate und somit adäquater auffassen. Die Referenz auf Bachtin - der seine Theorie anhand von Prosa entwickelt - wirft zugleich die Frage nach literaturwissenschaftlichen Konsequenzen auf.

Eine realweltliche große Bandbreite an Möglichkeiten des kreativen Sprachgebrauchs sollte nicht mit deren direkter Abbildung in literarischen Texten verwechselt werden. Vielmehr ist aus literaturwissenschaftlicher Sicht zu unter- 
suchen, inwiefern poetische Verfahren bereits der Vorstellung von Sprachen als Konglomerate Rechnung tragen. Im Sinne dieser Argumentation wird hier der Roman En egen strand [Ein eigener Strand] ${ }^{1}$ der schwedischen Autorin Aino Trosell (2013) als Fallbeispiel gelesen. Der Roman handelt von der Reise der Schwestern Hanna und Judit, die Schweden den Rücken kehren und sich auf einer nicht näher identifizierbaren Pazifikinsel permanent niederlassen wollen. Der Kontakt mit der lokalen Bevölkerung wird dabei größtenteils auf Englisch wiedergegeben. Außerhalb dieser eher touristischen Kommunikationssituationen verwenden Hanna und Judit ebenso untereinander ab und an Englisch. Die Implikationen dieser Heteroglossie jenseits der Authentizitätsvergewisserung stehen im Mittelpunkt der Analyse, die Mehrsprachigkeit als Index für Intertextualität untersucht. Es wird eine von der neueren soziolinguistischen Forschung angeregte Perspektive auf literarische Texte erprobt, die das gemeinsame Interesse beider Disziplinen an Formen und Varianten der Sprachverwendung in den Vordergrund stellt.

\section{Literaturwissenschaftliche Zugänge zur Mehr- sprachigkeit in der Moderne}

Die skizzierte Entwicklung hin zu einer Auffassung von Sprachgebrauch als Nutzung kommunikativer Ressourcen in der Soziolinguistik findet ein literaturwissenschaftliches Pendant in der Arbeit Yasemin Yildiz' (2012). In ihrer Monographie Beyond the Mother Tongue. The Postmonolingual Condition untersucht Yildiz mit der deutschen Sprache arbeitende Autorinnen und Autoren des 20. Jahrhunderts. Dabei wird das Korpus nicht zwangsläufig in einem metaphorischen ,Jenseits“ („,beyond“) verortet, sondern gemäß des vielzitierten Untertitels die Zentralität einer postmonolingual condition für die Literaturproduktion der Gegenwart herausgestellt. Nach Yildiz $(2012,2)$ etablierte sich im 18. Jahrhundert ein monolinguales Paradigma, das durch eine bis heute populär nachwirkende Homologie von Sprache, Ethnizität, Kultur und Nation gekennzeichnet sei. In der Moderne hingegen begänne sich ein Bewusstsein um diese Homologie herauszubilden, sodass im Sinne des Präfixes ,post' fortan gegen dieses Paradigma literarisch angeschrieben werden könne. Yildiz $(2012,5)$ betont zugleich einen außerliterarischen Rahmen, wenn sie die Bedeutung alltäglicher multilingualer Praktiken hervorhebt, um die postmonolingual condition zu charakterisieren. Ihre

1 Alle Übersetzungen von Zitaten aus dem Roman stammen vom Autor des vorliegenden Aufsatzes. 
Argumentation weist damit Parallelen zu Buschs soziolinguistischer Auffassung auf, da literarisches Schreiben unter dem Aspekt des kreativen Einsatzes sprachlicher Ressourcen untersucht wird.

Mehrsprachigkeit in literarischen Texten kann mit Yildiz als modernistische Auflehnung gegen ein sozial etabliertes monolinguales Paradigma gelesen werden. Yildiz' Verweis auf die Anwendung von Mehrsprachigkeit im Alltag könnte jedoch im Fall von Aino Trosells (2013) En egen strand dazu verleiten, die zeitweilige Kommunikation auf Englisch als einfache Wiedergabe der ,Realität‘ $\mathrm{zu}$ verstehen. $\mathrm{Zu}$ Beginn des 21. Jahrhunderts scheint es wenig verwunderlich, dass die Schwestern Hanna und Judit bei einem Auslandsaufenthalt ihre kommunikativen Ressourcen frei einsetzen.

Die Englischkenntnisse von Hanna und Judit fallen dem Leser zunächst nicht weiter auf, da die beiden Angehörige der kosmopolitisch orientierten Mittelschicht Schwedens darstellen sollen. Im soziolinguistischen Sprachgebrauch könnte dies als „enregisterment“ verstanden werden. Mit dem Begriff bezeichnet Asif Agha (2005, 38) die stereotype Zuordnung von Sprecherinnen und Sprechern zu sozialen Gruppen anhand verwendeter Sprachstile. Es scheint somit einigermaßen Konsens über die Normalität von Mehrsprachigkeit zu herrschen, wenn Sprachstile in erster Linie als Ausdruck sozialer Gruppenzugehörigkeit identifizierbar sind. Verliert Mehrsprachigkeit als stilistisches Mittel in literarischen Texten dadurch ihr kritisches Potenzial?

Während die Mehrsprachigkeit in En egen strand aus zeitgenössischer Perspektive relativ alltäglich erscheint, lässt sich mit literaturgeschichtlichem Hintergrundwissen ein Traditionsbewusstsein des Romans erkennen. Trosells Roman ruft die Themen auf, die die Literaturwissenschaftlerin Juliette Taylor-Batty (2013) in ihrer Monographie Multilingualism in Modernist Fiction ${ }^{2}$ als typisch für modernistische englischsprachige Autorinnen und Autoren um 1900 nennt, die Mehrsprachigkeit in literarischen Texten einsetzen: „exile, travel and intercultural encounter“ (Taylor-Batty 2013, 39). So begeben sich Hanna und Judit in ein selbstgewähltes Exil, wobei ihre Reise unvermeidlich zu interkulturellen Kontakten führt. Dieser Zugang zu Mehrsprachigkeit über bestimmte literarische Themen kann eine andere Perspektive anbieten, in der die textexterne Wirklichkeit nicht als direkter Referenzrahmen gilt. Stattdessen wird die Aufmerksamkeit auf intertextuelle Bezüge zu literarischen Traditionen der Medialisierung dieser Wirklichkeit gelenkt.

Taylor-Batty sieht den modernistischen Einsatz von Mehrsprachigkeit differenzierter als Yildiz. Mehrsprachigkeit wird im Modernismus nicht primär als

2 Ich danke Hannah Tischmann von der Universität Wien für diesen Literaturhinweis. 
widerständige Schreibpraktik gegen ein monolinguales Paradigma eingesetzt. Vielmehr handle es sich um eine Form der literarischen Verarbeitung des Modernisierungsschubes des 19. Jahrhunderts, wie aus Taylor-Battys Arbeit hervorgeht. Dabei ließen sich idealtypisch zwei extreme Positionen ausmachen: a) Zum einen gäbe es eine essentialistisch intendierte Reflektion von Mehrsprachigkeit und Kritik an Sprachmischungen. „In such texts, languages are often kept separate, their distinctiveness emphasised; interlingual interference, on the other hand, is presented with some ambivalence [...]“" (Taylor-Batty 2013, 37). b) Zum anderen gäbe es eine Tendenz zur experimentellen Auskostung gerade dieser Sprachmischungen.

In such texts, multilingualism troubles notions of linguistic, cultural and national identity and ,origins' through the productive stylisation of processes of interlingual interference, mixing and error, and in the use of translational processes that fundamentally undermine the traditional distinction between ,original' and ,translation', between source and target languages and cultures. (Taylor-Batty 2013, 37-38)

Yildiz fokussiert nur auf den zweiten Aspekt b) und lässt die erstere Variante a) unbeachtet. Im Fall von Variante a) werden Darstellungen von Mehrsprachigkeit jedoch als Argument für ein monolinguales Paradigma eingesetzt.

Das sich zwischen den beiden von Taylor-Batty identifizierten Positionen ergebene Spannungsfeld soll hier als Folie für die Analyse von En egen strand fruchtbar gemacht werden. Vor dieser Folie wird die Nuanciertheit des Einsatzes von Mehrsprachigkeit in Trosells Roman deutlich. In der folgenden Analyse wird argumentiert, dass Mehrsprachigkeit in En egen strand eine stilistische Funktion hat und einem sozialkritischen Kommentar auf die schwedische Gegenwartsgesellschaft dienen soll: 1) In einem ersten Analyseschritt werden mehrsprachige Szenen des Romans auf Verfahren der Authentizitätsvergewisserung hin analysiert. Dabei soll festgestellt werden, wie durch diese Verfahren zunächst der Eindruck eines einfachen ,Realismus' mit essentialistischen Untertönen hergestellt wird. Dieser Eindruck dient dem Roman als Grundlage seiner Kritik. 2) Anschließend soll in einem weiteren Schritt der Bezug des Romans auf den Modernismus aufgezeigt werden. Eine intertextuelle Verbindung zu Virginia Woolfs (2000 [1929]) Essay A Room of One's Own macht deutlich, dass En egen strand ästhetisch und thematisch auf die experimentelle Seite des Modernismus verweist und ein feministisches Programm verfolgt. Die Verwendung von Englisch kann so als Referenz auf den woolf'schen Prätext gelesen werden. 


\section{Verfahren der Authentizitätsvergewisserung}

Laut dem im Auftrag der Europäischen Union im Jahr 2012 erhobenen Eurobarometers können 53,97\% der schwedischen Bevölkerung Englisch. Damit liegt das Land im EU-weiten Vergleich an erster Stelle (vgl. Van Parys 2012). Angesichts dieser großen Anglophonie kann davon ausgegangen werden, dass der implizite Leser von schwedischsprachiger Literatur englische Begriffe in Texten problemlos versteht. Das entsprechende Phänomen, das Begriffe einer , anderen‘ Sprache in literarischen Texten keiner Form von Übersetzung bedürfen, bezeichnet der Komparatist Laurence Sternberg (1981) als „vehicular matching“. Sternberg hat in seinem bereits älteren Aufsatz „Polylingualism as Reality and Translation as Mimesis“ eine relativ umfassende Terminologie zur Beschreibung von mehrsprachigen Phänomenen in literarischen Texten entwickelt. Zur besseren Unterscheidung von realweltlichen, sozialen Phänomenen nennt er Mehrsprachigkeit in literarischen Texten ,polylingualism“ (Sternberg 1981, 222). Die Terminologie ist hilfreich, um Verfahren der Authentizitätsvergewisserung in literarischen Texten narratologisch sichtbar zu machen, wie die folgenden Beispiele zeigen.

In Vorbereitung auf ihren Inselaufenthalt kaufen die Schwestern Hanna und Judit bei einem lokalen Händler ein:

Vi pekar på hyllorna bakom honom [d.i. handlaren] på andra sidan disken, vi frågar och diskuterar. Hans engelska är dålig. [...] Han är inte påträngande, inte underdånig, inte inställsam. Och han verkar ha så god reda på vad vi vill ha att när slutsumman nämns, för säkerhets skull nerklottrad på en bit papper, prutar vi inte, även om vi lovat oss själva att självklart ska vi göra det. Men på svenskt vis tycker vi nu att det skulle framstå som snålt och dumt och att det skulle förstora den goda affärsmässiga relationen (Trosell 2013, 63). ${ }^{3}$

Zunächst verweist der erste Teil des Zitats auf die Kommunikationssituation. „Hans engelska är dålig“ ist dabei ein Fall von „explicit attribution“ (Sternberg 1981, 231), also der deutlichen Benennung der verwendeten Sprache. Im nächsten Absatz findet sich bereits eine weitere der von Sternberg $(1981,230)$ beschriebenen Varianten: die „conceptual reflection“. Hierbei werden die in einer anderssprachigen Gesprächssituation beiderseitig angewandten soziokulturellen Normen von

3 „Wir zeigen auf die Regale hinter ihm [d.i. der Händler] auf der anderen Seite des Tresens, wir fragen und diskutieren. Sein Englisch ist schlecht. [...] Er ist nicht aufdringlich, nicht unterwürfig, nicht schmeichlerisch. Und er scheint so gut zu wissen, was wir haben wollen, sodass wir, als er den Endpreis nennt, zur Sicherheit auf einen Zettel gekritzelt, nicht feilschen, obwohl wir uns das selbstverständlich vorgenommen hatten. Aber auf schwedische Art denken wir nun, dass wir geizig und dumm wirken würden, und dass es die gute Geschäftsbeziehung zerstören würde.“ 
der Erzählinstanz kommentiert und bewertet. Dadurch erscheinen entsprechende Passagen in En egen strand zunächst wie Anekdoten aus einem der Authentizität verpflichteten Reisebericht.

Die anekdotenhaft geschilderte Situation erhält im Text auf der nächsten Seite eine Pointe, als auf einmal ein Fall von „selective reproduction“ vorliegt und die Worte des Verkäufers angesichts der Erwägung eines scheinbar überflüssigen Kühlschrankkaufs wie folgt wiedergegeben werden: „In case you are interested it will be a pleasure for me to arrange the transport - within the price of course. Här har vi mannen som vet hur en slipsten ska dras, plötsligen på en överraskande god engelska!“ (Trosell 2013, 64). ${ }^{4}$ Im zweiten Zitat werden in umgekehrter Reihenfolge die Strategien „conceptual reflection“ und „explicit attribution“ verkürzt wiederholt, um die offensichtlich fälschlichen Vorannahmen der Erzählinstanz zu karikieren. Dies kann insofern als ein entscheidender Moment der Figurencharakterisierung angesehen werden, da ein eurozentrischer Blick auf den globalen Süden im Verlauf des Romans immer wieder von der Erzählinstanz direkt adressiert wird - die vorwiegend in den beiden Schwestern fokalisierte Erzählinstanz spricht ständig von exotisierendem Gebaren in einem kritischen Ton.

Interessanterweise führen die Charaktere das Gespräch in der zitierten Szene anschließend nicht auf Englisch weiter. Stattdessen erscheint eine neue Figur aus dem Nichts, die für den weiteren Handlungsverlauf entscheidend ist. Es handelt sich um einen Mann, der unter Rückgriff auf exotisierende Stereotype als gut aussehend beschrieben und für die Schwestern zum sexuellen Begehrensobjekt wird. Das Begehren veranlasst letztlich den Kühlschrankkauf (vgl. Trosell 2013, 64). Dieser Situationsverlauf zeigt eine deutliche Kluft zwischen der Rhetorik der Erzählinstanz und ihrem Verhalten. Im Kontext des Einsatzes von Mehrsprachigkeit als Authentizitätsmarker ist diese Szene bedeutend: Anstatt der Wiedergabe eines englischsprachigen Kaufgesprächs wird die Handlung mittels eines Deus ex Machina vorangetrieben. Die Erfüllung von Authentizitätserwartungen liegt an dieser Stelle im Roman eher fern.

Dem analysierten Verkaufsgespräch lassen sich bereits viele Informationen über die privilegierte soziale Stellung der Hauptfiguren im globalen Kontext entnehmen. Hanna und Judit gehören der weißen kosmopolitischen Mittelschicht Schwedens an und sind sich der Vorteile ihrer Position und der Gefahren von Vorurteilen bewusst. Wenn die zwei miteinander sprechen, bedienen sie sich gerne des ,svengelskas“ [„Schwenglisch“], einer humoristischen Mischform des Schwe-

4 „In case you are interested it will be a pleasure for me to arrange the transport - within the price of course. Hier erleben wir einen Mann, der zu überzeugen weiß, plötzlich mit einem überraschend guten Englisch.“ 
dischen und Englischen. Sternbergs (1981, 223) Begriff des „vehicular matching“ passt dazu nicht völlig, da die Streuung von englischem Vokabular hauptsächlich im Erzählen gegenüber dem Leser oder im Gespräch der Schwestern vorkommt, die Schwedisch zur Hauptsprache haben. Dieses Verhalten kann im Rahmen der Suche nach Verfahren der Authentizitätsvergewisserung als Variante des „enregisterments“ nach Agha $(2005,38)$ gewertet werden. Das Englische indiziert in diesem Sinne die Klassenzugehörigkeit. Sternberg (1981, 224) würde dieses Phänomen im Text vermutlich als „vehicular promiscuity“ klassifizieren, also die Darstellung von einsprachigen Situationen mit mehrsprachigen Mitteln. Es ist eine Strategie, die er mit der mittelalterlichen makkaronischen Dichtung in Verbindung bringt. Bei dieser Textform wird meist eine lingua franca parodiert und damit die sie verwendenden mächtigen Würdenträger bzw. allgemein sozial besser gestellte Personen. Besonders deutlich wird diese Facette in einem Rollenspiel der beiden Schwestern:

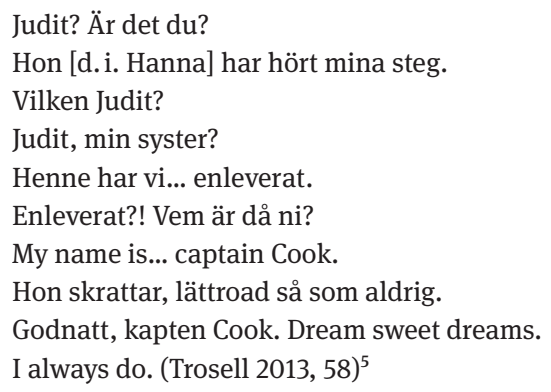

Mit der Anspielung auf Kapitän James Cook wird spielerisch die - im Text eindeutig mit Anglophonie assoziierte - koloniale Vergangenheit aufgegriffen. Die englischen Fragmente verweisen somit in diesem Kontext nicht nur auf die Klassenzugehörigkeit. Zugleich scheint es der Erzählinstanz schwerzufallen, sich von dieser Vergangenheit völlig zu distanzieren. Der Humor geht auf die Kosten von

5 ,Judit? Bist du es?

Sie [d.i. Hanna] hat meine Schritte gehört.

Welche Judit?

Judit, meine Schwester?

Sie haben wir... entführt.

Entführt? Wer seid dann ihr?

My name is... captain Cook.

Sie lacht, leicht zu amüsieren wie immer.

Gute Nacht, Kapitän Cook. Dream sweet dreams.

I always do.“ 
Hanna und Judit. Sie müssen sich als Europäerinnen zu den Mächtigen im pazifischen Archipel zählen und deshalb werden sie selbstironisch zum Ziel ihres eigenen Spottes.

Es gilt aufgrund der Einsicht über die Privilegien sozusagen ,you är ju även du“ [you heißt halt auch du], wie gegen Ende des Romans festgestellt wird. Im globalen Maßstab spielt es keine Rolle, ob Schweden im Gegensatz zu England eine starke Kolonialgeschichte hatte oder nicht. Dies ist allerdings nur einer der identitätspolitischen Konflikte, die im Roman verhandelt werden. Der Ausspruch fällt nach dem dramatischen Höhepunkt der Handlung: Judit und Hanna müssen von der Insel mittels eines selbstgebauten Floßes fliehen. Auf dem offenen Meer begegnen sie dem Kühlschranklieferanten. Dieser wird von Hanna bei einem Streit um dessen Kanu erschlagen. Kurz danach verstirbt Judit und Hanna versucht nach der Rettung, an Land auch deren Leiche verschwinden zu lassen. Letztlich findet sie sich in dem Ort wieder, wo die hier bereits erwähnte Einkaufsszene stattfand.

Die Protagonistin Hanna plagen nach der Rückkehr in das Dorf ihres Opfers gravierende Zweifel an der eigenen Identität. Die Identitätskrise der Protagonistin bezieht sich nur hintergründig auf ihre privilegierte soziale Stellung. „You är ju även du, han [d.i. handlaren] menade kanske bara mig, som om hon [d.i. Judit] inte existerade då, när vi provianterade hos honom. Det var alltid mig han vände sig till“ (Trosell 2013, 256). ${ }^{6}$ Nach dieser Selbsterkenntnis und ersten Spur versichert 13 Seiten später ein Zeitungsartikel, dass sich in der Diegese keine zwei Schwestern auf eine Pazifikinsel zurückgezogen hätten. Von den Behörden wurde stattdessen nur eine Person als vermisst gemeldet. Hanna scheint unter Schizophrenie zu leiden und Judit war eine Facette ihrer Persönlichkeit. Die von TaylorBatty (2013) identifizierte ambivalente Position a) gegenüber Mehrsprachigkeit in modernistischen literarischen Texten könnte in diesem Krankheitsbild zunächst vermutet werden. Zugleich läuft diese Wendung den Verfahren der Authentizitätsvergewisserung und damit verbundenen Formen des „enregisterments“ nach Agha $(2005,38)$ zuwider. Die Enthüllung Judits als krankhafte Einbildung stellt den vermeintlichen ,Realismus“ in jedem Fall rückblickend in Frage. Wie können die Erlebnisse einer Figur erzählt werden, die in der erzählten Welt nicht wirklich existiert? Und haben die englischen Passagen als Verfahren der Authentizitätsvergewisserung lediglich die Funktion, die Irritation des Lesers zu steigern?

6 „You heißt halt auch du, er [d.i. der Händler] meinte vielleicht nur mich, als ob sie [d.i. Judit] nicht existierte, als wir bei ihm proviantierten. Es war immer ich, an die er sich wendete.“ 


\section{Mehrsprachigkeit als intertextueller Verweis}

Der Einsatz von Englisch in Trosells Roman En egen strand hat eine indexikalische Funktion abseits der Verfahren der Authentizitätsvergewisserung. Die ,andere“ Sprache im Erzählen kann als allgemeiner intertextueller Verweis auf englischsprachige Literatur gelesen werden. Es findet im Roman eine Positionierung zu diesem ,fremden' und dennoch vertrauten Kanon statt. So tritt nicht nur der für seine Forschungsreisen im Pazifik weltberühmte James Cook in En egen strand auf. Judit vergleicht die Lage der beiden Schwestern ebenso mit Daniel Defoes Robinson Crusoe (vgl. Trosell 2013, 67).

Abgesehen von expliziten Verweisen auf englische Prätexte kann die Wahl einer Insel zum Handlungsort als weitere Referenz interpretiert werden. Die Insel wird im Modernismus als imaginärer Ort verstanden, der als Grundlage für die Umsetzung künstlerischer Projekte dient, wie die Literaturwissenschaftlerin Jill Franks $(2006,18)$ in ihrer Monographie Islands and the Modernists: The Allure of Isolation in Art, Literature and Science herausstellt. Hanna folgt dieser Idee und reist in den Pazifik. Der utopische Gedanke einer Autonomie des künstlerischen Subjekts treibt die Protagonistin an, ihr altes Leben hinter sich zu lassen. Einen Rückblick auf ihre Kindheit und ihre prekäre Arbeit im Sozialdienst schließt sie entsprechend mit dem Ausruf „Allt för konsten!“7 (Trosell 2013, 171) ab. In derselben Passage deutet sie zugleich an, Sprache vor allem als Instrument des subjektiven Ausdrucks von Individualität zu verstehen. Nach einem Beispiel dafür, wie sehr sie ihren beruflichen Jargon verinnerlicht hat, fragt sie: „När ska jag hitta tillbaka till mitt eget språk?“ (Trosell 2013, 171). ${ }^{8}$

Der modernistische Wunsch nach Autonomie des Subjekts hat wie die expliziten Anspielungen auf den Kolonialismus einen konkreten englischen Prätext. Bereits der Titel des Romans, En egen strand, erinnert nicht nur zufällig an den Titel der schwedischen Übersetzung von Virginia Woolfs (2000 [1929]) Klassiker A Room of One's Own. Statt Ett eget rum [Ein eigener Raum] ist es ein Strand für sich allein, der das Ausleben der eigenen Kreativität ermöglichen soll. Das künstlerische Streben nach Autonomie und die Machtasymmetrien des Kolonialismus stehen jedoch konträr zueinander. Im Roman wird die Auflösung dieses Widerspruchs versucht, indem sich mit Woolf auf eine geschlechterpolitische Problemlage berufen wird. Nach Woolf (2000 [1929], 67) wäre materielle Unabhängigkeit Voraussetzung für Kreativität, aber Frauen würde beides von den sozialen Umständen nicht gewährt.

7 „Alles für die Kunst!“

8 „Wann werde ich zu meiner eigenen Sprache zurückfinden?“ 
Der materielle Reichtum der Schwedin Hanna im Kontext des globalen Südens ist zwar immer schon überformt von dem im Roman durch Nennung von James Cook und Defoes Robinson Crusoe ,männlich“ kodierten Projekt des Kolonialismus. Dennoch wird eine geschlechtersensible Position vertreten, die auf den sozialen Ausschluss von Frauen aus ästhetischen Traditionen aufmerksam machen will. Die intertextuellen Bezüge zu Woolfs Essay in der Erzählung verdeutlichen dies: Die aktive Künstlerin unter den beiden ist nicht Hanna, sondern Judit. Ihr Name verweist auf den woolf'schen Prätext, denn „Judith“ heißt William Shakespeares historisch nicht-existente Schwester, die Woolf zum Zwecke ihrer Argumentation erfindet. Während diese Gemeinsamkeit noch zufälliger Art sein könnte, so erinnert das Schicksal der Hauptfigur von Trosells Roman stark an jenes, das Woolf für Judith Shakespeare ausmalt: „[...] any woman born with a great gift in the sixteenth century would certainly have gone crazed, shot herself, or ended her days in some lonely cottage outside the village, half witch, half wizard, feared and mocked at“. (Woolf 2000 [1929], 63-64) Die anscheinende Schizophrenie der Hauptfigur ist nur eine konsequente Umsetzung von Woolfs Anregungen aus $A$ Room of One's Own. Dort formuliert Woolf den utopischen Gedanken, dass eine Gesellschaft angestrebt werden solle, in der das kreative Potenzial aller Menschen frei entfaltet werden könne. Im Schweden des 21. Jahrhunderts scheinen diese Voraussetzungen weiterhin nicht erfüllt, wie Trosells Roman kritisch nahelegt.

\section{Resumée}

Mit Blick auf Woolfs (2000 [1929]) Essay kann die vermeintliche Schizophrenie der Protagonistin von En egen strand als eine literarische Stellungnahme zum derzeitigen Diskurs über Mehrsprachigkeit und Heteroglossie in der Soziolinguistik und Literaturwissenschaft interpretiert werden. Die von Taylor-Batty (2013) für den Modernismus identifizierten Extrempositionen zu Mehrsprachigkeit sind dabei aufschlussreich: a) Trotz psychologischer Probleme der Hauptfigur lässt der intertextuelle Verweis des Romans an der Vermutung zweifeln, dass eine mehrsprachigkeitskritische Position eingenommen wird; b) vielmehr wird stattdessen der Variante gefolgt, Unterschiede zwischen Original und Übersetzung zu verwischen. Dafür spricht die Anlehnung des Titels an Woolfs Prätext und die Übertragung der Figur Judit/Judith. Kultur wird somit in diesem Roman nicht mit einer Ethnie und einer spezifischen Sprache in eine Homologie überführt, sodass die Parameter der postmonolingual condition nach Yildiz (2012) erkennbar sind. Dennoch wird eine spezifisch auf die schwedische Gesellschaft ausgerichtete feministische Kritik in En egen strand formuliert. Der Zugang zu, in Buschs (2013) Terminologie, „,sprach- 
lichen und kommunikativen Ressourcen“ wird dabei im Roman einerseits wohlwollend aufgegriffen, und andererseits stilistisch eingesetzt, um geschlechtsspezifische Ausschlüsse in bestimmten kulturellen Feldern anzuprangern. Die Verwendung von Englisch in En egen strand verweist so nicht nur auf Verfahren der Authentizitätsvergewisserung und auf die Zweckmäßigkeit der Verwendung von Englisch als lingua franca in einer globalisierten Welt. Das fragmentarisch gebrauchte Englisch in En egen strand ist ebenso Index für den weltweiten Einfluss eines englischsprachigen, vom Kolonialismus geprägten Literaturkanons. Dieser Kanon wird dabei nicht ausschließlich negativ dargestellt. Der ebenfalls in diesem Kanon formulierte utopische Gedanke künstlerischer Autonomie wird vielmehr als Möglichkeitshorizont präsentiert. Als stilistisches Mittel wird das Englische in En egen strand mit idiolektaler Wirkung eingesetzt und dadurch die Suche der Hauptfigur nach einer individuell-eigenen künstlerischen Ausdrucksmöglichkeit unterstrichen. Das sich im Text darstellende Selbst der Hauptfigur charakterisiert sich implizit über die Heteroglossie seiner Äußerungen. Der Einsatz von Heteroglossie bietet Aino Trosells Roman ein Potenzial zur freien Gestaltung, dessen Bezugssystem in erster Linie literarisch ist. Zugleich macht die Analyse von En egen strand darauf aufmerksam, dass Mehrsprachigkeit im soziolinguistischen Sinne und literarische Heteroglossie bis zu einem gewissen Grad interdependent sind. Die Analyse des Zusammenspiels beider Seiten bietet sich aus literaturwissenschaftlicher Perspektive an. Die Mehrsprachigkeit von Gesellschaften ist nicht Vorlage, sondern produktive Grundlage für literarische Texte.

\section{Literaturverzeichnis}

Agha, Asif. „Voice, footing, enregisterment“. Journal of Lingustic Anthropology 15 (2005): 38-59.

Busch, Brigitta. Mehrsprachigkeit. Wien: Facultas.wuv, 2013.

Franks, Jill. Islands and the Modernists: The Allure of Isolation in Art, Literature and Science. Jefferson, NC: McFarland, 2006.

Sternberg, Meir. „Polylingualism as Reality and Translation as Mimesis“. Poetics Today 2.4 (1981): 221-239.

Taylor-Batty, Juliette. Multilingualism in Modernist Fiction. Basingstoke: Palgrave Macmillan, 2013.

Trosell, Aino. En egen strand. Stockholm: Norstedts, 2013.

Van Parys, Jonathan. A visualisation of language knowledge in Europe based on the European Commission's Europeans and their languages survey. Eurobarometer 2012, http:// languageknowledge.eu/countries/sweden, Interactive Database 2012- (05. Oktober 2017). Woolf, Virginia. A Room of One's Own. Oxford: Oxford Univ. Press, 2000 [1929].

Yildiz, Yasemin. Beyond the Mother Tongue: The Postmonolingual Condition. New York, NY: Fordham Univ. Press, 2012. 
Philipp Wagner, M.A. in Literatur- und Kulturtheorie (Universität Tübingen), zuvor Studium der Skandinavistik und Politikwissenschaft. Fellow der DFGgeförderten IRTG 1540 „Baltic Borderlands. Shifting Boundaries of Mind and Culture in the Borderlands of the Baltic Sea Region“ der Universität Greifswald. Forschungsschwerpunkte: Insularitäten, Mobilität, Mehrsprachigkeit, Intersektionalitätsansätze. 